\title{
DIFFERENTIAL ROLE OF N-TERMINAL POLYPROTEIN PROCESSING IN CORONAVIRUS GENOME REPLICATION AND MINIGENOME AMPLIFICATION
}

\author{
Carmen Galán, Luis Enjuanes, and Fernando Almazán*
}

\section{INTRODUCTION}

Processing of replicase polyproteins is a crucial step in the coronavirus life cycle, providing active components of the replication-transcription complex. ${ }^{1}$ Coronavirus ORF1a encodes one or two papain-like proteinases (PLPs) that are responsible for the cleavage at the N-proximal region of the replicase. Although PLP-1 cleavage products have been identified in $\mathrm{HCoV}-229 \mathrm{E}$ virus-infected cells, no specific roles or functional requirements have been studied so far for the HCoV-229E or TGEV proteins p9 (nsp1) and $\mathrm{p} 87$ (nsp2).

In the present study, we characterize by reverse genetics the effect of a point mutation at position 637, which mapped in the putative PLP-1 cleavage site at the p9/p87 junction, on TGEV and minigenome replication. A correlation was found between predicted cleaving and noncleaving mutations and the different nucleotides selected for virus or minigenome replication, respectively.

\section{MATERIALS AND METHODS}

\subsection{Plasmid Constructs and Recovery of Viruses from the cDNA Clones}

Point mutations at genome position 637 were engineered in $\mathrm{pBAC}-\mathrm{TGEV}^{\mathrm{FL}} 2 \mathrm{using}$ an overlapping PCR strategy. BHK cells stably transformed with the porcine aminopeptidase $\mathrm{N}$ gene were transfected with the cDNA clones using Lipofectamine 2000 (Invitrogen). After a 6-h incubation period at $37^{\circ} \mathrm{C}$, cells were trypsinized and plated over a confluent monolayer of swine testis (ST) cells. Cell supernatants were harvested for titration at 24,36 , and $48 \mathrm{~h}$ post-transfection.

\footnotetext{
* Centro Nacional de Biotecnología, CSIC, Darwin 3, Cantoblanco, 28049 Madrid, Spain.
} 
The cDNAs encoding TGEV-derived RNA minigenomes DI-C (9.7 kb) and M33 (3,3 kb) were previously described. ${ }^{3}$ The M33L minigenome was derived from the M33 minigenome including a 16-bp linker sequence. M33L and DI-C cDNAs with point mutations at position 637 were generated by restriction fragment exchange from plasmids with the corresponding mutations. For the trans-cleavage assay, the PLP-1 domain (Glu859 to Ser1315) from pp1a, and the N-terminal 610 amino acids with either Gly (637 G) or Asp (637 A) at position 108, were cloned into pcDNA3 (Invitrogen).

\subsection{Minigenome Rescue Quantification}

T7-driven in vitro transcripts of M33L minigenome mutants were transfected in ST cells previously infected with TGEV PUR46-MAD strain, and five serial passages were performed on fresh ST cells. Total RNA from each passage was used as template for realtime RT-PCR reactions (Q-RT-PCR) with minigenome-specific primers.

\subsection{Trans-Cleavage Assay}

TGEV PLP-1 proteinase and ppla substrates were expressed using the TNT T7coupled reticulocyte lysate system (Promega). Different enzyme to $\left[{ }^{35} \mathrm{~S}\right] \mathrm{Met}$-labeledsubstrate $[\mathrm{E} / \mathrm{S}]$ ratios were incubated overnight at $30^{\circ} \mathrm{C}$. Cleavage reactions were resolved by SDS-10\% PAGE and processed for fluorography.

\section{RESULTS AND DISCUSSION}

\subsection{Mutations at Genome Position 637 Severely Affect rTGEV Recovery}

During the construction of the TGEV full-length cDNA clone, a point mutation that was present in the defective minigenome DI-C (637-T) was maintained as a genetic marker. Interestingly, while other markers remained stable in the recombinant virus rescued (rTGEV), nucleotide 637 reproducibly reverted to the parental virus sequence $\mathrm{G}$ or $\mathrm{C}$, indicating a strong selective pressure at this position.

To study the role of nucleotide 637 in virus replication, TGEV cDNA mutants at this position were generated. Nucleotide substitution of the viral sequence $G$ at position 637 by $\mathrm{C}, \mathrm{T}$, or A produces an amino acid change at position 108 of the ppla from Gly to Ala, Val, or Asp, respectively. Virus recovery efficiency (Fig. 1A) and plaque morphology (Fig. 1B) were analyzed after cDNA transfection. No differences between the viruses carrying a $\mathrm{G}$ or $\mathrm{C}$ at position 637 were detected. However, a reduction of three logarithmic units and small plaque morphology was observed for viruses with $\mathrm{T}$ or $\mathrm{A}$ at position 637. Mutations that severely affected virus recovery from the cDNA correlated with more drastic amino acid substitutions. No correlation was found between RNA secondary structure predictions of the mutants and virus phenotypes (data not shown), indicating that the effect of mutations at position 637 was most likely at the protein level.

\subsection{Effect of Mutations at Position 637 on Minigenome Rescue Efficiency}

To study the sequence requirements at position 637 for minigenome replication, M33L minigenome mutants at this position were generated, and their rescue efficiency 
was determined by Q-RT-PCR (Fig. 1C). Controls of RNA transfection (RNA input) and background levels (Mock; TGEV; NTC) were included. Only M33L minigenomes with A or $\mathrm{T}$ at position 637 were efficiently rescued, in contrast with the sequence requirements at the same position for efficient virus recovery from the full-length cDNA clone. A late increase in the RNA accumulation observed for M33L-637G and C was due to a genotypic reversion at position 637 to A. No differences in virus titers that could explain minigenome phenotypes were observed (data not shown). The same mutational analysis with the RNA DI-C $(9.5 \mathrm{~Kb})$ showed identical results to that of M33L $(3.3 \mathrm{~Kb})$ indicating that the same sequence requirements at nucleotide 637 were necessary for both DI-C and M33L minigenome amplification regardless of their differences in size (data not shown).

A

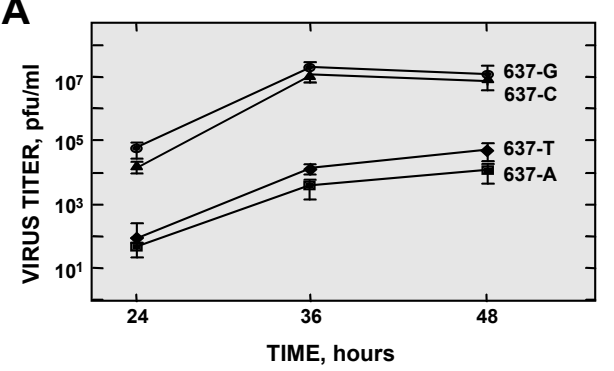

B

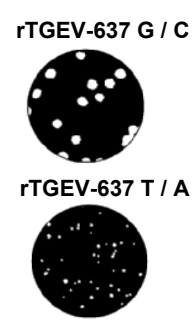

C

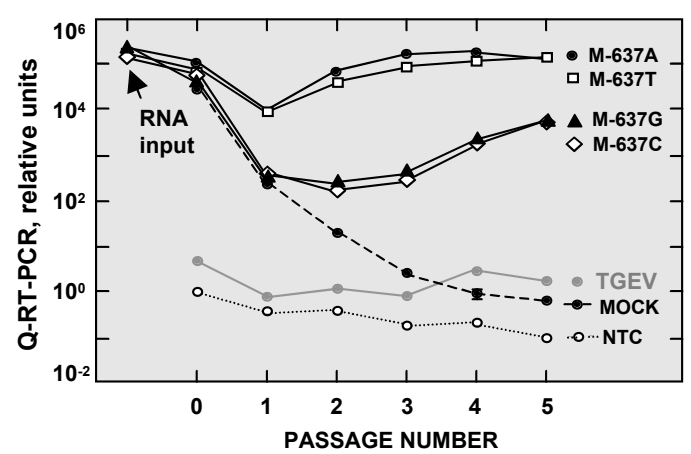

Figure 1. Phenotypes of rTGEV viruses or minigenome mutants. Effect of mutations at position 637 on virus rescue (A), virus plaque morphology (B), and minigenome amplification (C).

\subsection{Mutations at Nucleotide 637 Affect N-terminal Replicase Processing by PLP-1}

Sequence alignments reported for the HCoV-229E and TGEV replicase polyproteins, proposed two potential N-terminal cleavage sites in the TGEV ppla, ${ }^{4}$ although no experimental data have been provided to date. Mutations at nucleotide 637 affects the nature of amino acid 108 that occupies the $\mathrm{P} 1$ ' or $\mathrm{P} 3$ residues relative to the Thr107/Gly108 or Gly110/Ala111 putative cleavage sites, respectively, according with 
A

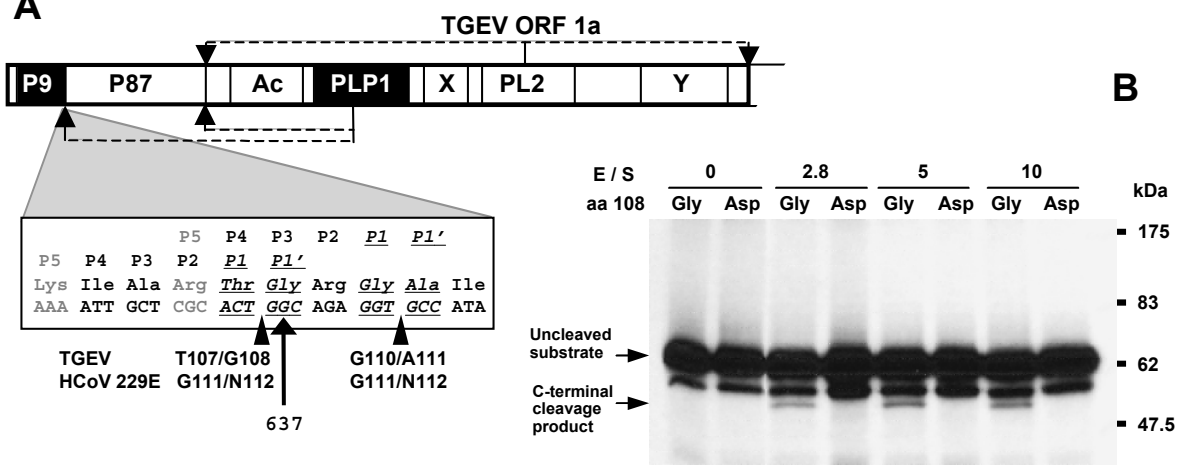

Figure 2. Effect of point mutations at nucleotide 637 on N-terminal replicase processing. (A). Scheme showing the two possible N-terminal cleavage sites (black arrowheads) predicted by sequence alignment with the HCoV$229 \mathrm{E}$ and the position of nucleotide 637. (B). Trans-cleavage assay.

these predictions (Fig. 2A). In both cases, a drastic amino acid change at position 108 could affect PLP1- mediated processing.

To support this hypothesis a trans-cleavage assay was performed with the TGEV PLP-1 domain and a N-terminal ppla substrate with either the wild-type sequence G at position 637 or an $\mathrm{A}$ at the same position, leading to the least conservative amino acid change (Gly108Asp) (Fig. 2B). In the absence of enzyme, only the 67-kDa substrate and a minor protein, probably resulting from a premature termination event, were detected. When the PLP-1 was included, a processed form of the substrate appeared with the substrate containing a Gly residue at position 108, with the expected size of the Cterminal cleavage product generated after the release of the N-terminal $\mathrm{p} 9$ protein, but not with the substrate presenting an Asp residue at the same position. These results indicated that mutations at nucleotide 637 affected the PLP-1-mediated N-terminal polyprotein cleavage in vitro. Because predicted cleaving mutations were required for virus recovery, we propose a critical role of this processing event for viral replication. In contrast, the minigenomes selected predicted noncleaving mutations, suggesting that the processing of the minigenome-encoded fusion protein led to the generation of ppla products that interfere with minigenome amplification. Further analysis will be required to assign specific functions to the $\mathrm{N}$-terminal replicase proteins in TGEV replication.

\section{REFERENCES}

1. J. Ziebuhr, E. J. Snijder, and A. E. Gorbalenya, Virus-encoded proteinases and proteolytic processing in the Nidovirales, J. Gen. Virol. 81:853-879 (2000).

2. F. Almazán, J. M. González, Z. Pénzes, et al., Engineering the largest RNA virus genome as an infectious bacterial artificial chromosome, Proc. Natl. Acad. Sci. USA 97, 5516-5521 (2000).

3. A. Izeta, C. Smerdou, S. Alonso, et al., Replication and packaging of transmissible gastroenteritis coronavirus-derived synthetic minigenomes, J. Virol. 73:1535-1545 (1999). 
4. J. Herold, A. E. Gorbalenya, V. Thiel, B. Schelle, and S. G. Siddell, Proteolytic processing at the amino terminus of human coronavirus 229E gene 1-encoded polyproteins: identification of a papain-like proteinase and its substrate, J. Virol. 72:910-918 (1998). 